\title{
VALIDAÇÃO DE METODOLOGIA ANALÍTICA PARA DETERMINAÇÃO DE MERCÚRIO TOTAL EM AMOSTRAS DE URINA POR ESPECTROMETRIA DE ABSORÇÃO ATÔMICA COM GERAÇÃO DE VAPOR FRIO (CV-AAS). ESTUDO DE CASO
}

\author{
Sabine Neusatz Guilhen, Maria Aparecida Faustino Pires e Elizabeth Sonoda Keiko Dantas* \\ Centro de Química e Meio Ambiente, Instituto de Pesquisas Energéticas e Nucleares, Universidade de São Paulo, Av. Lineu Prestes, \\ 2242, 05508-000 São Paulo - SP, Brasil \\ Fernanda Villibor Xavier \\ Fundação de Medicina Tropical do Tocantins, Av. Dionísio Farias, 838, 77814-350 Araguaína - TO, Brasil
}

Recebido em 31/8/09; aceito em 1/2/10; publicado na web em 3/5/10

\begin{abstract}
VALIDATION OF AN ANALYTICAL METHOD FOR THE DETERMINATION OF TOTAL MERCURY IN URINE SAMPLES USING COLD VAPOR ATOMIC ABSORPTION SPECTROMETRY (CV-AAS). CASE STUDY. Mercury is a toxic metal used in a variety of substances over the course history. One of its more dubious uses is in dental amalgam restorations. It is possible to measure very small concentrations of this metal in the urine of exposed subjects by the cold vapor atomic absorption technique. The present work features the validation as an essential tool to confirm the suitability of the analytical method chosen to accomplish such determination. An initial analysis will be carried out in order to evaluate the environmental and occupational levels of exposure to mercury in 39 members of the auxiliary dental staff at public consulting rooms in the city of Araguaína (TO).
\end{abstract}

Keywords: validation; mercury amalgam; CV-AAS.

\section{INTRODUÇÃO}

O mercúrio é um metal tóxico empregado em uma variedade de produtos e processos. Ocorre naturalmente no ambiente e existe sob as formas metálica ou elementar, inorgânica e orgânica. O mercúrio elementar é um metal líquido à temperatura ambiente, inodoro, de cor prateada e aparência brilhante. Com a exceção dos gases nobres, é o único elemento cujo vapor é monoatômico à temperatura ambiente. ${ }^{1}$

O vapor de mercúrio é facilmente absorvido pelo corpo devido a sua lipossolubilidade, mas seu tempo de vida é bastante limitado por ser rapidamente oxidado de mercúrio elementar $\left(\mathrm{Hg}^{0}\right)$ a mercúrio mercúrico $\left(\mathrm{Hg}^{2+}\right)$. $\mathrm{O}$ íon $\mathrm{Hg}^{2+}$ liga-se a grupamentos sulfidrila $(-\mathrm{SH})$ e seleno-hidrila (-SeH) de proteínas e enzimas. Alguns complexos Hg-SH são capazes de mimetizar moléculas endógenas dos sítios de ligação de proteínas na membrana plasmática e de sítios de ligação de proteínas e enzimas intracelulares. ${ }^{2}$

O mercúrio elementar $\left(\mathrm{Hg}^{0}\right)$ é solúvel em gorduras (lipossolúvel), o que lhe permite atravessar membranas celulares e ser absorvido pelo organismo. A absorção ocorre principalmente pelas vias respiratória e digestiva. A primeira é, indubitavelmente, a mais importante via de introdução no organismo para o vapor de mercúrio elementar, pois este é absorvido do ar e se difunde rapidamente através da membrana alvéolocapilar, passando para o sangue e estabelecendo-se nos eritrócitos. ${ }^{1,3}$

Segundo a Organização Mundial da Saúde, ${ }^{4}$ o teor de mercúrio total urinário considerado normal (pessoas não expostas) é de até 4 $\mu \mathrm{g} \mathrm{L}{ }^{-1}$. No Brasil, a Norma Regulamentadora 7 (NR-7), ${ }^{5,6}$ que dispõe sobre os parâmetros do controle biológico da exposição ocupacional a agentes químicos, propõe a utilização do mercúrio urinário total como indicador biológico da exposição (IBE) de trabalhadores ao mercúrio e seus compostos. Estabelece ainda o valor de $50 \mu \mathrm{g} \mathrm{L}^{-1}$ como índice biológico máximo permitido de mercúrio total urinário e $10 \mu \mathrm{g} \mathrm{L}^{-1}$ como valor de referência indicando normalidade.

Uma aplicação um tanto polêmica do mercúrio é no amálgama dental, uma liga composta de mercúrio $\left(\mathrm{Hg}^{0}\right)$, prata $\left(\mathrm{Ag}^{0}\right)$, estanho

*e-mail: esdantas@ipen.br
$\left(\mathrm{Sn}^{0}\right)$, cobre $\left(\mathrm{Cu}^{0}\right)$ e, possivelmente, outros elementos metálicos como o zinco $\left(\mathrm{Zn}^{0}\right) .{ }^{7}$ É crescente a preocupação com os riscos à saúde decorrentes da exposição ao vapor de mercúrio liberado na preparação do amálgama dental em consultórios odontológicos. ${ }^{8}$ Entretanto, existem poucas evidências para sustentar ou refutar inteiramente tais alegações.

Recentemente, a Fundação de Medicina Tropical do Tocantins iniciou um estudo para avaliar os níveis de exposição ambiental e ocupacional ao mercúrio no atendimento odontológico de consultórios públicos de Araguaína. Em colaboração com este estudo, o Laboratório de Análises Químicas e Ambiental do Centro de Química e Meio Ambiente do Instituto de Pesquisas Energéticas e Nucleares (IPEN) se responsabilizou pela realização das análises das amostras de urina dos indivíduos expostos quanto ao teor de mercúrio total por espectrometria de absorção atômica com vapor frio (CV-AAS). Para tanto, foi necessário primeiramente definir uma metodologia analítica adequada e validá-la para garantir a qualidade dos resultados.

Neste contexto, portanto, este estudo representa uma etapa prévia às análises propriamente ditas e tem por objetivo validar a metodologia analítica a ser empregada na determinação de mercúrio total em amostras de urina por CV-AAS.

O desempenho da metodologia analítica foi testado quanto aos seguintes parâmetros: limite de detecção e limite de quantificação, seletividade, linearidade, exatidão e precisão. Os ensaios foram realizados com material de referência certificado, o que assegura a rastreabilidade dos resultados.

\section{PARTE EXPERIMENTAL}

Na literatura, é possível encontrar variadas metodologias para determinação de mercúrio total em urina. ${ }^{9-12}$ A metodologia analítica foi selecionada da literatura com base na estrutura do laboratório analítico e dos recursos à disposição do analista.

A metodologia adotada foi descrita por Lopez-Colon e Lozano ${ }^{10}$ e consiste na determinação de mercúrio total em amostras de urina por espectrometria de absorção atômica com geração de vapor frio. 


\section{Materiais}

Tubos de polipropileno de $15 \mathrm{~mL}(120 \times 17 \mathrm{~mm})$ da Sarstedt, micro e macropipetas automáticas Wheaton Socorex Calibra, béqueres e balões volumétricos foram utilizados na preparação dos reagentes.

\section{Reagentes}

Os reagentes utilizados foram: material de referência certificado (MRC) de mercúrio em água (SRM 1641d; 1,590 $\pm 0,018 \mathrm{mg} \mathrm{kg}^{-1}$ ) e de elementos tóxicos em urina liofilizada (SRM 2670a; 95,1 \pm 0,98 $\mu \mathrm{g}$ $\mathrm{L}^{-1}$ ), ambos do National Institute of Standards and Technology (NIST, Gaithersburg, MD, USA), Triton ${ }^{\otimes} \mathrm{X}-100\left(\mathrm{C}_{33} \mathrm{H}_{60} \mathrm{O}_{10,5}\right)$ p.a. da Merck, dicromato de potássio $\left(\mathrm{K}_{2} \mathrm{Cr}_{2} \mathrm{O}_{7}\right)$ da CAAL Reagentes Analíticos, ácido nítrico $65 \%\left(\mathrm{HNO}_{3}\right)$ p.a. da Merck, ácido sulfúrico 95-97\% $\left(\mathrm{H}_{2} \mathrm{SO}_{4}\right)$ p.a. da Merck, permanganato de potássio $\left(\mathrm{KMnO}_{4}\right)$ p.a. da Merck, boro-hidreto de sódio $\left(\mathrm{NaBH}_{4}\right)$ p.a. da Merck, ácido clorídrico $37 \%(\mathrm{HCl})$ p.a. da Merck, hidroxilamina clorídrica $\left(\mathrm{NH}_{2} \mathrm{OH} . \mathrm{HCl}\right)$ p.a. da Nuclear, hidróxido de sódio $(\mathrm{NaOH})$ p.a. da Merck e uma solução padrão de mercúrio em $\mathrm{HNO}_{3} 5 \%\left(1000 \mathrm{mg} \mathrm{L}^{-1}\right)$ da Merck.

\section{Equipamentos}

Utilizou-se um espectrômetro de absorção atômica PerkinElmer AAnalyst 800 acoplado a um sistema PerkinElmer de injeção em fluxo FIAS 400, cujas condições de operação estão descritas na Tabela 1, uma balança Gehaka BG400 e uma estufa da Ética.

Tabela 1. Condições operacionais para a análise de Hg por CV-AAS

\begin{tabular}{|c|c|c|c|c|c|}
\hline Variáveis & \multicolumn{5}{|c|}{ Condições de operação } \\
\hline Tempo de integração (s) & \multicolumn{5}{|c|}{20} \\
\hline Lâmpada & \multicolumn{5}{|c|}{ EDL de $\mathrm{Hg}$} \\
\hline $\begin{array}{l}\text { Comprimento } \\
\text { de onda }(\mathrm{nm})\end{array}$ & \multicolumn{5}{|c|}{253,7} \\
\hline Slit (nm) & \multicolumn{5}{|c|}{0,7} \\
\hline \multirow[t]{2}{*}{$\begin{array}{l}\text { Temperatura } \\
\text { da célula }\left({ }^{\circ} \mathrm{C}\right)\end{array}$} & \multicolumn{5}{|c|}{100} \\
\hline & Etapa & $\begin{array}{l}\text { Tempo } \\
\text { (s) }\end{array}$ & $\begin{array}{l}\text { Bomba 1 } \\
\left(\mathrm{U} \mathrm{min}^{-1}\right)\end{array}$ & $\begin{array}{l}\text { Bomba } 2 \\
\left(\mathrm{U} \mathrm{min}^{-1}\right)\end{array}$ & Válvula \\
\hline \multirow[t]{3}{*}{$\begin{array}{l}\text { Velocidade } \\
\text { da bomba }\end{array}$} & Prefill & 15 & 100 & 120 & Fill \\
\hline & 1 & 10 & 100 & 120 & Fill \\
\hline & 2 & 15 & 0 & 120 & Inject \\
\hline
\end{tabular}

\section{Soluções}

As soluções utilizadas foram: Triton ${ }^{\circledR} \mathrm{X}-100$ 0,2\% (v/v); $\mathrm{HNO}_{3}$ $50 \%(v / v) ; \mathrm{K}_{2} \mathrm{Cr}_{2} \mathrm{O}_{7} 0,5 \%(\mathrm{~m} / \mathrm{v}) ; \mathrm{KMnO}_{4} 5 \%(\mathrm{~m} / \mathrm{v})$; solução redutora $\mathrm{NaBH}_{4} 0,2 \%$ e NaOH $0,05 \%(\mathrm{~m} / \mathrm{v}) ; \mathrm{HCl} 3 \%(v / v) ; \mathrm{NH}_{2} \mathrm{OH} . \mathrm{HCl} 10 \%$ $(\mathrm{m} / \mathrm{v})$; solução diluente $\mathrm{HNO}_{3} 10 \%$ e $\mathrm{H}_{2} \mathrm{SO}_{4} 20 \%(v / v)$; e solução estoque de $500 \mu g \mathrm{~L}^{-1} \mathrm{de} \mathrm{Hg}(v / v)$, preparada a partir de uma solução padrão de mercúrio em $\mathrm{HNO}_{3} 5 \%\left(1000 \mathrm{mg} \mathrm{L}^{-1}\right)$ da Merck. A água utilizada no preparo das soluções foi do tipo ultrapura destilada 18,3 $\mathrm{M} \Omega \mathrm{cm}^{-1}$.

\section{Etapa experimental}

O procedimento analítico pode ser dividido em três etapas distintas: digestão, oxidação com permanganato de potássio $\left(\mathrm{KMnO}_{4}\right) \mathrm{e}$ redução com boro-hidreto de sódio $\left(\mathrm{NaBH}_{4}\right)$. A execução cuidadosa de cada uma destas etapas determina a qualidade dos resultados obtidos no processo como um todo.

\section{Digestão da amostra de urina}

A um tubo de polipropileno com capacidade para $15 \mathrm{~mL}$ foram adicionados: $1 \mathrm{~mL}$ de urina, $0,5 \mathrm{~mL}$ de solução de Triton ${ }^{\circledR} \mathrm{X}-100$ 0,2\% $(v / v), 0,1 \mathrm{~mL}$ de solução estabilizadora $\mathrm{K}_{2} \mathrm{Cr}_{2} \mathrm{O}_{7} 0,5 \%$ em $\mathrm{HNO}_{3} 50 \%$ $(\mathrm{m} / \mathrm{v}), 1 \mathrm{~mL}$ de $\mathrm{HNO}_{3}$ e $2 \mathrm{~mL}$ de $\mathrm{H}_{2} \mathrm{SO}_{4}$. A mistura foi então aquecida com o tubo fechado à temperatura constante de $60{ }^{\circ} \mathrm{C}$, pelo período de $12 \mathrm{~h}$ (overnight), para digestão da amostra.

As perdas de mercúrio foram evitadas ao máximo por meio da adição de dicromato de potássio e Triton ${ }^{\circledR}$. A solução de dicromato de potássio em meio ácido oxida o mercúrio, mantendo-o em solução na forma de íons $\mathrm{Hg}^{2+}$. A solução de Triton ${ }^{\circledR}$ adicionada previne perdas devido à ação bacteriana. A urina é uma amostra biológica e, portanto, sujeita à ação de bactérias que convertem os compostos de mercúrio solúveis em compostos voláteis de mercúrio. Ambos os reagentes, portanto, evitam perdas por vaporização. Além disso, o aquecimento foi feito em tubo fechado, o que dificulta ainda mais as perdas por vaporização.

\section{Oxidação com $\mathrm{KMnO}_{4}$}

Após a digestão, a solução deve ser retirada da estufa e deixada à temperatura ambiente para esfriar e, em seguida, $\mathrm{KMnO}_{4} 5 \%(\mathrm{~m} / \mathrm{v})$ deve ser adicionado em quantidade suficiente para que a solução adquira uma cor púrpura, indicando o excesso do oxidante em solução. Se a cor da solução persistir por cerca de $15 \mathrm{~min}$, significa que o $\mathrm{KMnO}_{4}$ está em excesso, o que garantirá a completa oxidação de todos íons de mercúrio em solução a $\mathrm{Hg}^{2+}$. A reação de oxidação do mercúrio elementar $\left(\mathrm{Hg}^{0}\right)$ a mercúrio mercúrico $\left(\mathrm{Hg}^{2+}\right)$ ou mercúrio (II) pelo $\mathrm{KMnO}_{4}$ é mostrada na Equação 1.

$3 \mathrm{Hg}_{\text {(aq) }}+2 \mathrm{KMnO}_{4(\mathrm{aq})}+\mathrm{H}_{2} \mathrm{O}_{\text {(1) }} \rightarrow 3 \mathrm{HgO}_{\text {(aq) }}+2 \mathrm{MnO}_{2 \text { (aq) }}+2 \mathrm{KOH}_{\text {(aq) }}$

A reação de oxidação do íon mercuroso $\left(\mathrm{Hg}_{2}{ }^{2+}\right)$ ou mercúrio (I) ao íon $\mathrm{Hg}^{2+}$ pelo $\mathrm{KMnO}_{4}$ é mostrada na Equação 2 .

$\underset{3 \mathrm{Hg}_{2} \mathrm{O}_{\text {(aq) }}}{2 \mathrm{KOH}_{(\text {aq) }}}+2 \mathrm{KMnO}_{4(\text { aq) }}+\mathrm{H}_{2} \mathrm{O}_{(\mathrm{l})} \rightarrow 6 \mathrm{HgO}_{(\text {aq) }}+2 \mathrm{MnO}_{2 \text { (aq) }}+$

Após $1 \mathrm{~h}$ em repouso, adicionam-se $15 \mu \mathrm{L}$ de hidroxilamina colorídrica $10 \%(\mathrm{~m} / \mathrm{v})$ para reduzir o excesso de permanganato em solução. A solução então readquire seu aspecto inicial (incolor). Por fim, a solução é diluída a um volume final de $10 \mathrm{~mL}$ com solução diluente.

\section{Redução com $\mathrm{NaBH}_{4}$}

A solução contendo íons $\mathrm{Hg}^{2+}$ é introduzida por injeção em fluxo e direcionada ao compartimento de reação, onde será reduzida a $\mathrm{Hg}^{0}$ pela solução redutora de boro-hidreto de sódio $(\mathrm{m} / \mathrm{v})$ e uma solução carregadora de ácido clorídrico 3\% (v/v). A reação entre o $\mathrm{NaBH}_{4} \mathrm{e}$ o $\mathrm{HCl}$ gera os seguintes produtos:

$\mathrm{NaBH}_{4(\mathrm{aq})}+3 \mathrm{H}_{2} \mathrm{O}_{(\mathrm{l})}+\mathrm{HCl}_{(\mathrm{aq})} \rightarrow \mathrm{H}_{3} \mathrm{BO}_{3(\mathrm{aq})}+\mathrm{NaCl}_{(\mathrm{aq})}+8 \mathrm{H}_{(\text {aq) }}^{*}$

A espécie $\mathrm{H}^{*}$ gerada reduz os íons $\mathrm{Hg}^{2+}$ na solução da amostra:

$\mathrm{nHg}^{2+}{ }_{(\mathrm{aq})}+\mathrm{H}_{(\mathrm{aq})(\text { excesso) }}^{*} \rightarrow \mathrm{nHg}_{(\mathrm{g})}^{0}+\mathrm{H}_{2(\mathrm{~g})(\text { excesso) }}$

$\mathrm{O}$ vapor de $\mathrm{Hg}^{0}$ é então conduzido por um gás de arraste (argônio, Ar) para o compartimento da amostra, posicionado no caminho óptico do espectrômetro, e quantificado.

\section{Validação analítica}

O desempenho da metodologia analítica é influenciado essencialmente pela qualidade das medidas instrumentais e pela confiabilidade estatística dos cálculos envolvidos no tratamento dos dados. ${ }^{13}$ 
Segundo a ANVISA, ${ }^{14}$ a validação deve garantir, através de estudos experimentais, que a metodologia atenda às exigências das aplicações analíticas, assegurando a confiabilidade dos resultados. O desempenho da metodologia analítica sob investigação precisa ser consistente com a aplicação para a qual ela se destina. ${ }^{15}$

A validação analítica é um processo demorado, que requer um grande número de ensaios e cálculos estatísticos, aumentando o custo das análises. Portanto, é necessário selecionar os parâmetros que têm o maior impacto sobre a qualidade dos resultados e a rapidez com a qual são obtidos. Por isso, muitas vezes, não é economicamente pertinente realizar todos os ensaios de validação sugeridos nos guias encontrados na literatura..$^{14,16-20}$

Os parâmetros selecionados no presente estudo para testar o desempenho da metodologia adotada ${ }^{10}$ foram: curva analítica, faixa de trabalho e faixa linear de trabalho, linearidade, seletividade, limite de detecção, limite de quantificação, exatidão e precisão.

\section{Curva analítica}

A curva analítica é a representação gráfica da relação entre a resposta obtida (y) e a concentração de analito (x). A maneira mais simples de descrever esta dependência é através do modelo de regressão linear representado por uma equação matemática (equação da reta) em que y está em função de x:

$$
y=a x+b
$$

O ajuste da curva pelo método dos mínimos quadrados fornecerá os coeficientes de regressão que, no caso de um ajuste linear, correspondem ao intercepto da reta com o eixo y ou coeficiente linear (b) e a inclinação da reta ou coeficiente angular (a).

A relação matemática entre $\mathrm{x}$ e y é previamente determinada empiricamente a partir de soluções de concentrações conhecidas de analito (ou padrões analíticos), dando origem à curva analítica. ${ }^{21}$

\section{Faixa de trabalho, faixa linear e faixa linear de trabalho}

A faixa de trabalho representa o intervalo de concentração no qual o método de determinação de mercúrio será aplicado. O limite inferior da faixa de trabalho, normalmente, é estipulado pelos valores dos limites de detecção (LD) e quantificação (LQ) do método. A faixa de trabalho adotada neste estudo compreende um intervalo de concentrações de 0 a $12 \mu \mathrm{g} \mathrm{L} \mathrm{L}^{-1}$.

A faixa de concentrações na qual a sensibilidade pode ser considerada constante é a faixa linear.

A faixa linear de trabalho é estabelecida através da confirmação de que o método é capaz de fornecer um grau aceitável de exatidão, precisão e linearidade quando aplicada às amostras contendo quantidades de analito dentro da faixa de trabalho especificada. Nesta faixa, portanto, o sinal medido (y) terá uma relação linear com a concentração do analito (x).

\section{Linearidade}

A linearidade corresponde à capacidade do método de fornecer resultados linearmente correlacionados às concentrações do analito em uma determinada faixa de aplicação. ${ }^{18,22}$ A Lei de Beer estabelece que a relação entre o sinal medido (y) e a concentração do analito (x), descrita pela Equação 1, só é válida para o intervalo correspondente à faixa linear da curva analítica.

A adequação do ajuste da curva é fornecida pelo coeficiente de correlação de Pearson, r, também conhecido como coeficiente de correlação produto-momento ou, simplesmente, $r$ de Pearson.

O coeficiente de correlação apresenta uma faixa de magnitude entre -1 e $1(-1 \leq \mathrm{r} \leq 1)$ e indica o quanto a reta pode ser considerada adequada como modelo matemático. ${ }^{16}$ Quanto mais próximo de 1 ou
-1, menor a dispersão do conjunto de pontos experimentais, ou seja, menor o erro em y. Nestas condições, o ajuste da função matemática escolhida para a curva analítica será máximo.

Um coeficiente de correlação maior que 0,999 é considerado como evidência de um ajuste ideal dos dados para a linha de regressão. ${ }^{23-25}$ A ANVISA ${ }^{14}$ recomenda um coeficiente de correlação maior ou igual a 0,99 e o INMETRO ${ }^{16}$ considera um valor de $\mathrm{r}$ superior a 0,90 como sendo o valor usualmente requerido. O método será considerado livre de tendências (unbiased) se a reta contiver a origem. Portanto, a obtenção de um $r$ superior a 0,9 assegura a linearidade da relação entre o sinal medido (y) e a concentração (x) para o intervalo de concentrações em questão.

A correlação entre as variáveis de estudo, em termos dos valores de $r$, pode ser interpretada conforme a Tabela 2 .

Tabela 2. Correlação entre as variáveis de estudo, em termos dos valores de r. Adaptada da ref. 26

\begin{tabular}{lc}
\hline Valor de $\mathrm{r}$ & Tipo de correlação \\
\hline $\mathrm{r}=1$ & Perfeita \\
$0,91<\mathrm{r}<0,99$ & Fortíssima \\
$0,61<\mathrm{r}<0,91$ & Forte \\
$0,31<\mathrm{r}<0,60$ & Média \\
$0,01<\mathrm{r}<0,30$ & Fraca \\
$\mathrm{r}=0$ & Nula \\
\hline
\end{tabular}

\section{Seletividade}

A matriz da amostra pode conter componentes que interferem no sinal analítico de interesse. A seletividade avalia o grau de interferência destes componentes sobre o sinal medido. A resposta obtida deve corresponder exclusivamente ao analito. ${ }^{27}$

A seletividade foi avaliada para a metodologia empregada, comparando-se dois grupos de amostras: um contendo a matriz (urina) e outro, no qual a matriz está ausente, ambos com concentrações de analito idênticas em cada nível de concentração de interesse.

O material de referência certificado $1641 \mathrm{~d}$ da NIST foi empregado como padrão de mercúrio, assegurando a rastreabilidade dos resultados obtidos. Alíquotas distintas deste material foram adicionadas aos dois grupos de amostras (com e sem urina) de forma a se obter três diferentes concentrações da faixa de trabalho: 1,59; 4,77 e 7,95 $\mu \mathrm{g} \mathrm{L}^{-1}$. O número de amostras paralelas (n) em cada nível de concentração correspondeu a 7, conforme recomendação do INMETRO. ${ }^{16}$

Para avaliar os resultados obtidos, aplicaram-se os testes F de Fisher-Snedecor e o t de Student.

\section{Limite de quantificação e limite de detecção}

Medir a capacidade de detecção e quantificação é particularmente útil quando o analito que se deseja mensurar está presente em quantidades muito baixas na amostra. Para validar um método analítico, é suficiente indicar a concentração do analito a partir da qual a quantificação e a detecção se tornam problemáticas.

O limite de quantificação (LQ) ou mínimo valor quantificável, por sua vez, é expresso em termos da concentração correspondente à precisão desejada (10\%), levando-se em consideração o desvio padrão relativo (DPR\%) calculado para uma determinada faixa de concentração. O limite de detecção (LD) ou mínimo valor detectável considera os riscos $\alpha$ (falso positivo) e $\beta$ (falso negativo).

\section{Limite de quantificação}

O limite de quantificação (LQ) é a menor concentração de analito que pode ser determinada com um nível aceitável de precisão e veracidade. O LQ pode ser obtido a partir da análise de 7 replicatas do branco da amostra: ${ }^{16}$ 


$$
\mathrm{LQ}=\mathrm{X}+\left(\mathrm{k}_{\mathrm{Q}}\right) \cdot \mathrm{s}
$$

O fator de multiplicação $\mathrm{k}_{\mathrm{Q}}$ é dependente da precisão (DPR\%) desejada no limite de quantificação.

$$
\operatorname{DPR}(\%)=100 / \mathrm{k}_{\mathrm{Q}}
$$

Por exemplo, se a precisão ou DPR\% desejada no limite de quantificação for de $10 \%, \mathrm{k}_{\mathrm{Q}}$ será igual a 10 .

\section{Limite de detecção}

O limite de detecção (LD) representa a mínima concentração ou quantidade de um analito que produz um sinal que o analista consiga distinguir, a um nível de confiança especificado, do sinal produzido pelo branco.

O LD do método foi calculado com base no desvio padrão obtido para uma série de 7 (n) replicatas do branco da amostra (matriz na qual o analito está ausente ou presente em concentrações muito pequenas) adicionadas com a menor concentração aceitável do analito. Os valores de desvio padrão obtidos são então aplicados na seguinte fórmula: ${ }^{16}$

$$
\mathrm{LD}=\mathrm{X}+\left(\mathrm{k}_{\mathrm{D}}\right) \cdot \mathrm{s}
$$

onde, X é a média dos valores obtidos para cada amostra adicionada, $\mathrm{k}_{\mathrm{D}}$ é o fator de multiplicação correspondente ao t de Student para um nível de confiança de $(1-\alpha) \times 100 \%$ e $(n-1)$ graus de liberdade e s corresponde ao desvio padrão calculado.

\section{Exatidão}

É improvável que um resultado obtido experimentalmente seja exatamente igual ao valor de referência convencionalmente aceito como verdadeiro. ${ }^{16,18,28}$ A exatidão (accuracy) é o grau de concordância entre o resultado de uma medição e um valor verdadeiro do mensurando. ${ }^{29}$ A exatidão do método foi avaliada a partir da realização de ensaios com material de referência certificado e testes de recuperação do analito.

A interpretação dos resultados obtidos pode ser feita com auxílio de algumas ferramentas matemáticas como o erro relativo, o teste $\mathrm{F}$, o índice Z (Z Score) e o erro normalizado.

A recuperação ou fator de recuperação $(\mathrm{R})$ é definida como a proporção da quantidade da substância de interesse, presente ou adicionada na amostra, recuperada por meio do método analítico. ${ }^{21}$ Reflete, portanto, a quantidade de determinado analito efetivamente quantificada em relação à quantidade "real" presente na amostra.

Quando a recuperação é obtida a partir da análise de material de referência certificado (MRC), calcula-se:

$$
\mathrm{R} \%=\left(\mathrm{C} / \mathrm{C}_{\mathrm{MRC}}\right) \cdot 100
$$

onde: $\mathrm{C}$ é a média das recuperações obtidas para $\mathrm{n}$ repetições e $\mathrm{C}_{\mathrm{MRC}}$ é a concentração verdadeira. O material de referência certificado 2670a da NIST foi empregado na avaliação da exatidão do método.

\section{Precisão}

A precisão avalia a dispersão dos resultados entre ensaios independentes, repetidos de uma mesma amostra, amostras semelhantes ou padrões, em condições definidas ${ }^{16,18}$ e pode ser expressa pelo desvio padrão relativo (DPR\%), também conhecido como coeficiente de variação.

Para avaliar a precisão, o desvio padrão relativo foi calculado para uma série de 7 replicatas independentes do material de referência certificado 2670a da NIST, em 3 diferentes concentrações da faixa de trabalho.
De acordo com Huber, ${ }^{30}$ os métodos empregados para quantificar o analito em escalas macro requerem um DPR\% de 1 a $2 \%$. Para a análise de traços, é aceitável um DPR\% de até 30\%, dependendo da complexidade da amostra. A Tabela 3 mostra a relação entre a concentração do analito e a precisão esperada.

Tabela 3. Relação entre a concentração do analito e a precisão esperada. Adaptada da ref. 30

\begin{tabular}{lccc}
\hline $\begin{array}{l}\text { Porcentagem } \\
\text { de analito }(\%)\end{array}$ & $\begin{array}{c}\text { Proporção } \\
\text { de analito }\end{array}$ & Unidade & DPR $(\%)$ \\
\hline 100 & 1 & $100 \%$ & 1,3 \\
10 & $10^{-1}$ & $10 \%$ & 2,8 \\
1 & $10^{-2}$ & $1 \%$ & 2,7 \\
0,1 & $10^{-3}$ & $0,1 \%$ & 3,7 \\
0,01 & $10^{-4}$ & $100 \mathrm{ppm}$ & 5,3 \\
0,001 & $10^{-5}$ & $10 \mathrm{ppm}$ & 7,3 \\
0,0001 & $10^{-6}$ & $1 \mathrm{ppm}$ & 11 \\
0,00001 & $10^{-7}$ & $100 \mathrm{ppb}$ & 15 \\
0,000001 & $10^{-8}$ & $10 \mathrm{ppb}$ & 21 \\
0,0000001 & $10^{-9}$ & $1 \mathrm{ppb}$ & 30 \\
\hline
\end{tabular}

\section{RESULTADOS E DISCUSSÃO}

A linearidade foi investigada para as concentrações da faixa de trabalho para uma série de 6 pontos distribuídos no intervalo de 0 a $12 \mu \mathrm{g} \mathrm{L}$ L $^{-1}$ O coeficiente de correlação obtido foi igual a 0,998 . O método pode, portanto, ser considerado linear, pois o coeficiente de correlação obtido a partir da curva analítica é superior ao valor recomendado pela ANVISA ${ }^{14}(r \geq 0,99)$. Além disso, pode-se dizer que a correlação entre as variáveis x (concentração) e y (sinal obtido), de acordo com a Tabela 2, é fortíssima.

A seletividade foi testada para avaliar a influência dos efeitos da matriz sobre os resultados. Os resultados (em $\left.\mu \mathrm{g} \mathrm{L}^{-1}\right)$ para um número de amostras paralelas equivalente a 7 (n) encontram-se resumidos na Tabela 4. Pelos resultados obtidos podemos concluir que a matriz não tem um efeito significativo sobre a precisão do método na faixa de concentrações de estudo.

Para estimar o limite de quantificação, a precisão do método foi examinada para uma faixa de concentrações do analito de 0,1 a $1 \mu \mathrm{g}$ $\mathrm{L}^{-1}$ (primeiro ponto da curva analítica) e 7 replicatas para cada nível de concentração. A média (X) e o desvio padrão (s) foram calculados com base nos resultados obtidos para cada concentração e aplicados na equação do desvio padrão relativo (DPR\%):

$$
\mathrm{DPR} \%=(\mathrm{s} / \mathrm{X}) \cdot 100
$$

Com base nos valores de DPR\% calculados, foi possível decidir a concentração limite, isto é, a menor concentração de mercúrio que pode ser determinada com a precisão desejada que, no caso, corresponde a $10 \%$.

Tabela 4. Valores de DPR\% obtidos no estudo do LQ

\begin{tabular}{lccccccc}
\hline Concentração $\left(\mu \mathrm{g} \mathrm{L}^{-1}\right)$ & 1,0 & 0,8 & 0,6 & 0,4 & 0,3 & 0,2 & 0,1 \\
\hline $\operatorname{DPR}(\%)$ & 3 & 2 & 3 & 5 & 7 & 22 & 123 \\
\hline
\end{tabular}

De acordo com os valores de DPR\% apresentados na Tabela 4, pode-se atribuir à concentração de $0,3 \mu \mathrm{g} \mathrm{L} \mathrm{L}^{-1}$ a denominação de menor concentração aceitável do analito, pois é a menor concentração que pode ser determinada dentro da precisão desejada. O limite de quantificação (LQ) é, portanto, $0,3 \mu \mathrm{g} \mathrm{L} \mathrm{L}^{-1}$.

O limite de detecção foi calculado da seguinte forma:

$$
\mathrm{LD}=0+\left(\mathrm{k}_{\mathrm{D}}\right) \cdot \mathrm{s}
$$


onde o fator de multiplicação $\left(\mathrm{k}_{\mathrm{D}}\right)$ corresponde ao valor de $t$ de Student para um nível de confiança de $99 \%$ e 6 graus de liberdade $(\mathrm{t}=3,143)$ e s é o desvio padrão obtido para uma série de 7 (n) replicatas do branco da amostra adicionadas com a menor concentração aceitável do analito (LQ). O LD calculado foi igual a $0,07 \mu \mathrm{g} \mathrm{\textrm {L } ^ { - 1 }}$.

A precisão foi determinada a partir do desvio padrão relativo $(\mathrm{DPR} \%)$ calculado para uma série de medições $(\mathrm{n}=7)$ sucessivas do analito sob condições de repetitividade. O desvio padrão foi calculado para cada concentração investigada na faixa de trabalho. Todos os resultados ficaram abaixo do limite considerado como aceitável (até $20 \%$ ) para que se considere o método preciso. ${ }^{30}$ Pode-se concluir, portanto, que o método adotado é preciso.

A exatidão foi avaliada comparando-se os valores obtidos experimentalmente dos valores certificados a partir do estudo da recuperação. Também se calculou o erro relativo, o índice $\mathrm{Z}$ e o erro normalizado. Os resultados podem ser observados na Tabela 5. Podese observar que o erro é maior para as concentrações mais próximas aos extremos (inferior e superior) da curva analítica, como previsto pela lei de Beer. Com base nos valores de $\mathrm{Z}$ obtidos, pode-se afirmar que o método é capaz de gerar resultados satisfatórios, pois $|Z| \leq 2$ em todas as concentrações. ${ }^{16}$

O material de referência certificado 2670a da NIST foi empregado no ensaio da recuperação e os resultados obtidos para uma série de 7 medições independentes em cada nível de concentração são mostrados na Tabela 5. O t de Student foi calculado para avaliar a exatidão do método. O método pode ser considerado exato se o valor de t obtido estiver dentro do intervalo estabelecido pelo valor tabelado de t para ( $\mathrm{n}-1)$ graus de liberdade e nível de confiança de $99 \%$. Todos os valores calculados de $\mathrm{t}\left(\mathrm{t}_{\text {calculado }}\right)$ são inferiores ao valor de $\mathrm{t}$ tabelado $\left(\mathrm{t}_{\text {tabelado }}\right)$ em todos os níveis de concentração investigados. O método pode, portanto, ser considerado exato.

Tabela 5. Conjunto de resultados obtidos através dos ensaios de validação

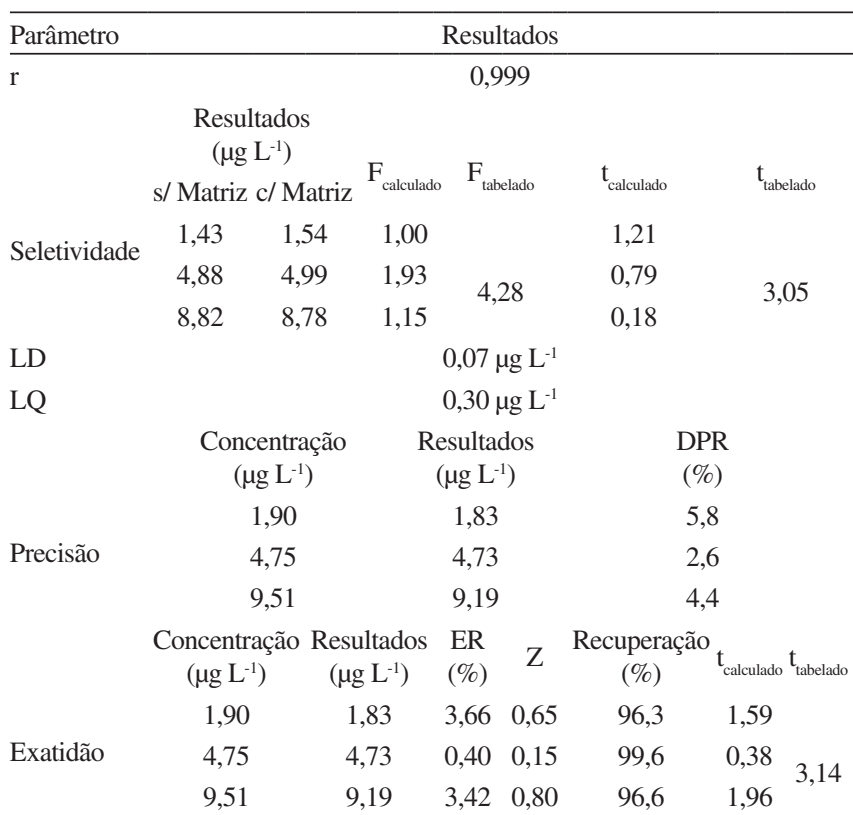

$\mathrm{r}=$ coeficiente de correlação; $\mathrm{LD}=$ limite de detecção; $\mathrm{LQ}=$ limite de quantificação

\section{Aplicação da metodologia em amostras reais de Tocantins}

A metodologia analítica foi aplicada em uma análise inicial para determinação de mercúrio total em 39 amostras de urina enviadas pela Fundação de Medicina Tropical do Tocantins. Estas amostras fazem parte de um estudo referente aos riscos da exposição ao mercúrio no atendimento odontológico de consultórios da rede pública da cidade de Araguaína (TO).

Do total de amostras analisadas, 6 (15\%) apresentaram níveis de mercúrio urinário superiores ao limite de normalidade equivalente a $10 \mu \mathrm{g} \mathrm{L}^{-1}$. A concentração obtida para uma das amostras ficou na concentração limiar $\left[(10,01 \pm 0,03) \mu \mathrm{g} \mathrm{L}^{-1}\right]$ e o resultado obtido para o restante das amostras ficou abaixo de $10 \mu \mathrm{g} \mathrm{L}^{-1}$, o que revela uma exposição ao mercúrio, ainda que dentro do limite de normalidade. A média dos resultados obtidos foi de $6 \mu \mathrm{g} \mathrm{L}^{-1} \mathrm{em}$ uma faixa de concentração de 1 a $23 \mu \mathrm{g} \mathrm{L}^{-1}$ com até $11 \%$ de precisão.

\section{CONCLUSÃO}

A validação analítica comprovou a adequação da metodologia adotada para quantificar $\mathrm{Hg}$ total em amostras de urina por espectrometria de absorção atômica com vapor frio. Todos os testes foram efetuados de modo a abranger a faixa de concentração na qual se pretende aplicar o método de determinação de mercúrio. A menor concentração de $\mathrm{Hg}$ que pode ser determinada com uma precisão aceitável corresponde a $0,30 \mu \mathrm{g} \mathrm{L}^{-1}$ (LQ). Além disso, o limite de detecção do método $\left(\mathrm{LD}=0,07 \mu \mathrm{g} \mathrm{L}^{-1}\right.$ ) assegura a determinação de $\mathrm{Hg}$ em quantidades traço. Entretanto, a relevância dos ensaios de validação recai sobre os parâmetros que confirmam a boa performance do método na faixa de aplicação. O desempenho satisfatório do método nesta faixa é essencial para que se possa extrair um significado bioquímico dos resultados, levando em conta os limites pré-estabelecidos. Os resultados devem possibilitar ao analista dizer se o teor de $\mathrm{Hg}$ urinário (HgU) determinado está ou não dentro da faixa de normalidade (até $10 \mu \mathrm{g} \mathrm{L}^{-1}$ ). Neste quesito, os testes para verificar a exatidão e a precisão do método foram fundamentais para demonstrar que os erros associados aos resultados não são estatisticamente significantes e que os mesmos são confiáveis. Além disso, a metodologia permite uma recuperação quase que total do analito investigado.

A metodologia adotada representa, portanto, uma importante ferramenta para avaliar a exposição ao $\mathrm{Hg}$ e os resultados obtidos constituem uma evidência de que esta exposição possa ter, de fato, alguma influência sobre os níveis de $\mathrm{Hg}$ no organismo. Ainda que direcionada à questão do amálgama dental, esta metodologia pode também ser aplicada para investigar outros casos nos quais exista uma exposição ao $\mathrm{Hg}$, salvo exposições aos compostos orgânicos de $\mathrm{Hg}$, para as quais a urina não é o IBE mais adequado. Espera-se, com isso, que o laboratório possa, futuramente, ampliar a sua área de atuação. Uma análise inicial permitiu constatar o teor de Hg urinário $(\mathrm{HgU})$ de 39 indivíduos pertencentes ao estudo da Fundação de Medicina Tropical do Tocantins, 6 dos quais apresentaram níveis de $\mathrm{HgU}$ superiores ao limite de normalidade. Os resultados obtidos encontram-se em uma faixa de concentração de 1 a $23 \mu \mathrm{g} \mathrm{L}^{-1}$ com até $11 \%$ de precisão. Testes adicionais deverão ser realizados para constatar se existe alguma alteração em função do tempo e da carga de exposição dos indivíduos aos quais correspondem as amostras analisadas.

\section{REFERÊNCIAS}

1. de Azevedo, F. A.; Toxicologia do mercúrio, RIMA: São Paulo, 2003.

2. Bridges, C. C.; Bauch, C.; Verrey, F.; Zalups, R. K.; J. Am. Soc. Nephrol. 2004, 15, 663 .

3. Fowler, B. A.; Nordberg, M.; Friberg, L.; Nordberg, G.; Handbook on the Toxicology of Metals, $3^{\text {rd }}$ ed., Academic Press: San Diego, 2007.

4. Campos, R. C.; Pivetta, F. Em ECO/OPS Série Vigilância; Câmara, V. de M., ed.; 1993, vol. 12, p. 129-136.

5. Brasil, Ministério do Trabalho; Normas Regulamentadoras de Segurança e Saúde no Trabalho, NR-7 - Norma Regulamentadora N ${ }^{\circ}$ 7, Anexo II, 
Portaria N 3214. Ministério do Trabalho e Emprego, Diário Oficial da União, 06/07/1978 (supl. 127): 24-25, disponível em http://www.saude. ba.gov.br/cesat/ColetaneaLegislacao/NormasRegulamentadoras_NRs / NR07.PDF>, acessada em Abril 2010.

6. Brasil, Ministério do Trabalho; Normas Regulamentadoras de Segurança e Saúde no Trabalho. NR-7 - Norma Regulamentadora № 7, Portaria N 24. Ministério do Trabalho e Emprego, Diário Oficial da União, 0/12/1994 (seção I): 21278-21280 em http://www.mte.gov.br/legislacao/ normas_regulamentaDORAS/nr_07_at.pdf, Dezembro 1994, acessada em Abril 2010.

7. World Health Organization; Mercury in Health Care, WHO, September 2005.

8. Skare, I.; Engqvist, A.; Arch Occup Environ Health, 1994, 49, 384.

9. https://www.varianinc.com/media/sci/apps/aa126.pdf, acessada em Abril 2010.

10. Lopez-Colon, J. L.; Lozano, R.; At. Spectrosc. 2006, 27, 98.

11. Moreton, J. A.; Delves, H. T.; J. Anal. At. Spectrom. 1998, 13, 659.

12. Tahán, J. E.; Granadillo, V. A.; Sánchez, J. M.; Cullibán, H. S.; Romero, R. A.; J. Anal. At. Spectrom. 1993, 8, 1005.

13. Ribeiro, F. A. de L.; Ferreira, M. M. C.; Quim. Nova 2008, 31, 164.

14. Brasil, Agência Nacional de Vigilância Sanitária; Resolução RE nº 899 , de 29 de maio de 2003, Diário Oficial da União, 02/06/2003.

15. EURACHEM/CITAC Guide; The Fitness for Purpose of Analytical Methods, EURACEM Working Group, December, 1998.

16. Instituto Nacional de Metrologia, Normalização e Qualidade Industrial; DOQ-CGCRE-008, Orientações sobre Validação de Métodos de Ensaios Químicos, Março, 2003.
17. Danzer, K.; Currie, L. A.; Pure Appl. Chem. 1998, 70, 993.

18. Guidance for Industry - Q2B Validation of Analytical Procedures: Methodology, European Agency for the Evaluation of Medical Products (ICH): Londres, 1996.

19. Pimentel, M. F.; Barros Neto, B.; Quim. Nova 1996, 19, 268.

20. Ribani, M.; Bottoli, C. B. G.; Collins, C. H.; Jardim, I. C. S. F.; Melo, L.; Quim. Nova 2004, 27, 771.

21. Thompson, M.; Ellison, S. L. R.; Wood, R.; Pure Appl. Chem. 2002, 74, 835.

22. Swartz, M. E.; Krull, I. S.; Pharm. Technol. 1998, 2, 12.

23. Green, J. M.; Anal. Chem. 1996, 68, 305A.

24. Jenke, D. R.; J. Chromatogr. Sci. 1998, 36, 179.

25. Shabir, G. A.; J. Chromatogr., A 2003, 987, 57.

26. Brito, N. M.; Amarante Junior, O. P. De; Polese, L.; Ribeiro, M. L.; R. Ecotoxicol. e Meio Ambiente 2003, 13, 129.

27. Vessman, J.; Stefan, R. I.; van Staden, J. F.; Danzer, K.; Lindner, W.; Burns, D. T.; Fajgelj, A.; Müller, H.; Pure Appl Chem. 2001, 73, 1381.

28. Leite, F.; Validação em Análise Química, $4^{\mathrm{a}}$ ed., Editora Átomo: Campinas, 2002.

29. Instituto Nacional de Metrologia, Normalização e Qualidade Industrial; Vocabulário internacional de termos fundamentais e gerais de metrologia (VIM), $3^{\text {a }}$ ed., Portaria INMETRO 029 de 1995: Rio de Janeiro, 2003.

30. Huber, L.; LC/GC International 2001, 11, 96. 